\title{
Minimum-energy packet forwarding policies for guaranteed LQG performance in wireless control systems
}

\author{
Zhenhua Zou, Burak Demirel and Mikael Johansson
}

\begin{abstract}
This paper studies minimum-energy packet forwarding policies for communicating sensor measurements from plant to controller over an unreliable multi-hop wireless network so as to guarantee that the optimal controller achieves a prespecified closed-loop performance. For fixed sampling interval, we demonstrate that the minimal linear-quadratic control loss is monotonically decreasing in the reliability of the sensor-to-controller communication. This allows us to decompose the overall design problem into two separate tasks: finding the minimum end-to-end reliability that allows to achieve a prespecified linear-quadratic loss, and developing minimum-energy packet forwarding policies under a deadlineconstrained reliability requirement. We develop optimal solutions for both subproblems and show how the co-designed system with minimum forwarding energy cost and guaranteed LQG control performance can be found by a one-dimensional search over admissible sampling periods. The paper ends with a numerical example which demonstrates the effectiveness of the proposed framework.
\end{abstract}

\section{INTRODUCTION}

Networked control systems (NCSs) are distributed systems that utilize communication networks to exchange information between system components such as sensors, controllers and actuators. Wireless communication promises advantages over wired communication in terms of increased flexibility and reduced wiring and maintenance costs, and is finding its way into a wide variety of applications (see [1] and the references therein). However, communication over an unreliable low-power wireless sensor network induces nonnegligible communication delays and packet losses which might have significant impact on the closed-loop performance. In particular, the closed-loop performance depends on the per-packet delivery delay and loss probability [2].

Although there is a vast literature on energy-efficient wireless communication, few papers target per-packet realtime communications suitable for analysis and design of networked control systems. Rate adaptation techniques that balance the transmission rate (which influence the per-packet latency) and the power consumption have been extensively studied (see e.g. [3]), but it is complex and costly to implement in wireless sensor networks. Duty cycling is the main technique to reduce energy consumption in wireless sensor network. Some recent work [4], [5] study duty cycle scheduling for maximizing the lifetime of a sensor network while guaranteeing an upper bound on the end-to-end packet delivery delay. An important observation from these papers is that the energy cost increases with the packet injection

The authors are with the ACCESS Linnaeus Center and the Automatic Control Lab, School of Electrical Engineering, KTH-Royal Institute of Technology, Sweden. (Email: \{zhenhua.zou, burak.demirel, mikaelj\}@ee.kth.se) rate (corresponding to the sampling rate of sensors) and the end-to-end delivery reliability. With this in mind, it is natural to account for the energy cost of the wireless network when designing wireless control system.

In this paper, we develop a co-design framework which allows to find the minimal energy cost for forwarding sensor packets over a multi-hop lossy wireless network while ensuring a targeted closed-loop control performance in terms of linear-quadratic loss. The co-design framework decomposes the overall design problem into two well-defined control and network subproblems, and the joint optimality can be proved by a similar monotonicity argument as in [2], [6]. In the control subproblem, we develop the optimal controller for fixed sampling interval, transmission delay and end-toend reliability and compute its associated linear-quadratic loss. By a monotonicity argument, the control performance constraint is transformed into a requirement on the minimum deadline-constrained reliability of sensor packets. The network subproblem then amounts to minimizing the forwarding energy cost subject to this requirement on the minimum probability that the packet is delivered within a hard deadline. The minimal energy cost and the associated optimal design is found by a one-dimensional search over sampling periods.

Some work has studied energy cost of the wireless network in a networked control system. Shi et.al [7] considered network lifetime maximization problems while satisfying desired estimation accuracy. Later, Mo et. al [8] have proposed a stochastic sensor scheduling algorithm to minimize the expected estimation error covariance matrix under given energy constraints. The most related work is by Park et. al [9]. They find critical protocol parameters to minimize the energy consumption subject to constraints on the packet loss probability and average delay computed from the desired control performance. While the overall aim is similar, the assumptions, solution techniques and optimality properties are distinctively different from ours. It considers the IEEE 802.15.4 MAC protocol in a star-topology network, heuristically replaces the control performance with the average delay, and cannot guarantee an optimal solution.

The paper is organized as follows. In Section II, we introduce models and assumptions. Section III formulates the minimum energy problem and describes a co-design framework that decomposes the design problem into well-defined control and network subproblems. Optimal solutions to these subproblems are presented in Section IV and Section V, respectively. Numerical examples are provided in Section VI. Finally, Section VII concludes the paper. 


\section{MODELS AND ASSUMPTIONS}

In this section, we detail the system model including the process and the wireless network, and introduce the assumptions and limitations of the controller architecture.

\section{A. Process model}

We consider the control of a stochastic linear system

$$
d x=A x d t+B u d t+d v_{c}
$$

where $x \in \mathbb{R}^{n}$ is the state, $u \in \mathbb{R}^{m}$ is the control signal, $A \in \mathbb{R}^{n \times n}$ and $B \in \mathbb{R}^{n \times m}$ are the system matrices, and $v_{c}$ is a Wiener process with incremental covariance $R_{v}^{c}$. We assume that a noisy measurement of the system output

$$
y(k h)=\tilde{C} x(k h)+w(k h)
$$

is taken every sample period $h$. Here $w(k h)$ is a discrete-time white noise Gaussian process, independent of the disturbance $v_{c}$, and with zero mean and covariance $R_{w}$. The sensor measurements are time-stamped and sent over an unreliable multi-hop network.

\section{B. Network model}

The network consists of a set of nodes $\mathcal{N}=\{1, \ldots, N\}$ that can receive and transmit data to the other nodes. We represent the network topology with a directed graph $\mathcal{G}=$ $(\mathcal{N}, \mathcal{L})$ where the presence of a link $(i, j) \in \mathcal{L}$ means that there is a non-zero probability that a packet transmitted by node $i$ can be received and correctly decoded by node $j$. Nodes are synchronized to a global clock and communication is slotted. Each slot is $t_{s}$ seconds long and allows for the transmission of a single packet and the reception of the associated acknowledgement from the receiver. We assume that packet losses on links are independent from losses on other links, and that losses on each link $(i, j) \in \mathcal{L}$ follow a Bernoulli process with loss probability $p_{i j}$. While the sensor data packets can be lost, we assume that acknowledgements can not. Nodes do not have access to channel state, only their statistics (i.e. the loss probabilities on the outgoing links).

The design problem for the network is to develop a forwarding policy $\pi$ that determines if a node should forward a received packet or drop it, and to which node it should attempt to transmit. We let $C(\pi)$ be the average energy cost per injected packet for packet forwarding policy $\pi$. Since the sensor injects a new packet once every $h$ seconds, the network energy cost is $C(\pi) / h$.

\section{Control architecture and control performance}

The controller and actuator nodes are assumed to be synchronized to the global clock and operate with a fixed lag $\tau \leq h$ relative to the sampling times of the sensor. The controller uses the information available at times $k h+\tau$ to compute the control action (and hence consider packets that have not arrived within $\tau$ seconds to be lost). The actuator uses zero-order-hold and maintains the same control action between the controller updates $[k h+\tau,(k+1) h+\tau)$ for $k=0,1, \ldots$ as seen in Figure 1 .

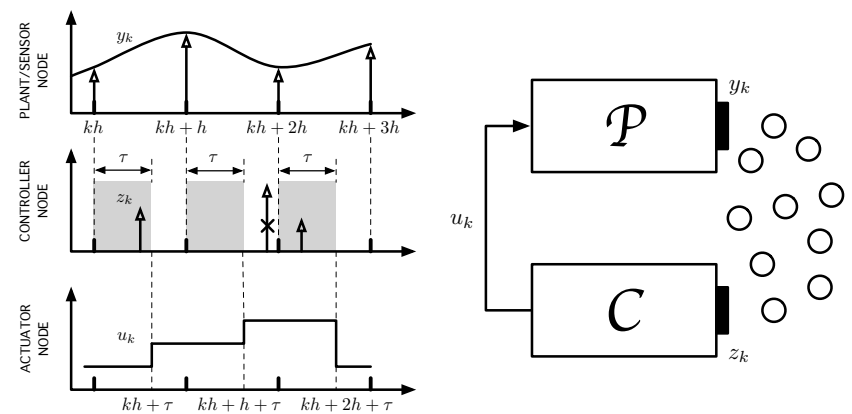

Fig. 1. Networked control system block scheme with timing diagrams for sensor, controller and actuator nodes.

Throughout this paper, we focus on co-design of the minimum-energy forwarding policy and the control law to guarantee a desired linear quadratic loss

$$
\begin{array}{r}
J=\mathbf{E}\left\{\int_{0}^{T}\left[\begin{array}{l}
x(t) \\
u(t)
\end{array}\right]^{\top}\left[\begin{array}{ll}
Q_{x x}^{c} & Q_{x u}^{c} \\
Q_{x u}^{c T} & Q_{u u}^{c}
\end{array}\right]\left[\begin{array}{l}
x(t) \\
u(t)
\end{array}\right] d t\right. \\
\left.+x^{\top}(T) Q_{0}^{c} x(T)\right\},
\end{array}
$$

where the matrices $Q_{x x}^{c}$ and $Q_{0}^{c}$ are symmetric and positive semi-definite while $Q_{u u}^{c}$ is symmetric and positive definite. The expectation is taken over the random process and measurement disturbances, as well as over the random packet losses in the network. Since the sampling rate is a design parameter in our system, it is critical to have a continuoustime loss function as basis of comparison.

\section{Minimum ENERG CO-DeSign PROBlem}

In the time-triggered control architecture described in Section II-C, only packets received within $\tau$ seconds are considered by the controller node. Let $\rho(\pi, h, \tau)$ be the probability that packets injected at rate $1 / h$ and forwarded using policy $\pi$ arrive at the controller within $\tau$ seconds. Hence, the problem becomes

$$
\begin{array}{ll}
\underset{h, \pi}{\operatorname{minimize}} & C(\pi) / h \\
\text { subject to } & J^{\star}(\tau, \rho(\pi, h, \tau), h) \leq J_{\text {req }},
\end{array}
$$

where $J^{\star}(\tau, \rho(\pi, h, \tau), h)$ is the optimal control loss under the packet delay $\tau$, packet loss probability $\rho$ and sampling period $h$. Furthermore, to limit the number of free parameters, we let the maximal latency $\tau$ equal the sampling period $h$. Finally, the problem we consider in this paper is

$$
\begin{array}{ll}
\underset{h, \pi}{\operatorname{minimize}} & C(\pi) / h \\
\text { subject to } & J^{\star}(\rho(\pi, h), h) \leq J_{\text {req. }} .
\end{array}
$$

Our design problem couples the sampling time selection, the design of the optimal packet forwarding policy, and the design of the control law in an apparently complex fashion. However, as we will show next, the optimal control loss has a monotonicity property that allows us to replace the control performance constraint with a constraint on the end-to-end reliability $\rho(\pi, h)$. Specifically, we have the following result: 
Theorem 3.1: For a given sampling interval $h$, the optimal control loss $J^{\star}(\rho, h)$ is monotone decreasing in the end-toend reliability $\rho$.

Proof: The proof relies on a coupling argument on the loss process for the end-to-end transmissions, see [6].

Using the monotonicity property of the control loss function $J^{\star}(\rho(\pi, h), h)$, we can replace the performance constraint $J^{\star}(\rho(\pi, h), h) \leq J_{\text {req }}$ with the reliability constraint $\rho(\pi, h) \geq \rho_{\min }(h)$, where $\rho_{\min }(h)$ is the unique end-to-end reliability for which $J^{\star}\left(\rho_{\min }(h), h\right)=J_{\text {req }}$. We have not been able to find an explicit formula for the inverse function of $J^{\star}$, but due to the monotonicity property, this value can be readily found by bisection.

By replacing the control performance constraint with the associated end-to-end reliability requirement, the design problem becomes

$$
\begin{array}{ll}
\underset{h, \pi}{\operatorname{minimize}} & C(\pi) / h \\
\text { subject to } & \rho(\pi, h) \geq \rho_{\min }(h) .
\end{array}
$$

For a fixed value of $h$, this problem amounts to finding the policy of minimum expected energy that guarantees that the probability that packets arrive at their destination within the deadline $h$ is greater than $\rho_{\min }$. An optimal solution to this problem will be given in Section V. If we let $C_{\min }(h)$ denote the minimum expected energy of the optimal policy for a given $h$, then original problem has been reduced to

$$
\underset{h}{\operatorname{minimize}} C_{\min }(h) / h \text {. }
$$

The optimal solution for this problem is simply found by sweeping over all admissible sampling periods $h$.

In summary, for a given $h$ we first compute the reliability requirement $\rho_{\min }(h)$ using bisection. Each step of the bisection algorithm requires finding the optimal controller and evaluating its performance as detailed in Section IV. When $\rho_{\min }(h)$ is found, the optimal forwarding policy and the associated minimum energy cost is found via a constrained Markov decision process detailed in Section V. The jointly optimal design is then found by a search over $h$.

\section{Control SubProblem}

The network delivers sensor packets with a fixed delay of $\tau$ seconds and losses with the probability $\rho_{\min }(h)$ for a given sampling interval $h$. We are interested in controllers that work on the information available at times $k h+\tau$, hold the control signal constant over intervals $[k h+\tau,(k+1) h+$ $\tau)$, and use control actions that are optimal in the sense of the linear-quadratic loss function (1).

For notational convenience, we assume that $T=N h$ for some integer $N>0$. Let $u_{k}=u(k h)$ be the control signal computed at time $k h+\tau$ and applied to the process during the time interval $[k h+\tau,(k+1) h+\tau)$ and let $x_{k}=x(k h)$. Then, the continuous-time loss function (1) can be transformed into an equivalent discrete-time loss

$$
J=\mathbf{E}\left\{\sum_{k=0}^{N-1}\left[\begin{array}{c}
\xi_{k} \\
u_{k}
\end{array}\right]^{\top}\left[\begin{array}{cc}
\Xi_{\xi \xi}^{c} & \Xi_{\xi u}^{c} \\
\Xi_{\xi u}^{c T} & \Xi_{u u}^{c}
\end{array}\right]\left[\begin{array}{c}
\xi_{k} \\
u_{k}
\end{array}\right]+\xi_{N}^{\top} \Xi_{0}^{c} \xi_{N}\right\}
$$

where $\Xi_{\xi \xi}^{c}, \Xi_{\xi u}^{c}, \Xi_{\xi u}^{c T}, \Xi_{u u}^{c}$ and $\Xi_{0}^{c}$ are given in [6].

The optimal control problem is then to compute the control sequence $\left\{u_{k}\right\}$ that minimizes the discrete-time loss function. The evolution of the system between sampling instants can be described in terms of the extended state vector $\xi_{k} \triangleq \operatorname{col}\left\{x_{k}, u_{k-1}\right\}$ as

$$
\begin{aligned}
& \xi_{k+1}=\underbrace{\left[\begin{array}{cc}
e^{A h} & \int_{h}^{h-\tau} e^{A s} d s B \\
0_{m \times n} & 0_{m \times m}
\end{array}\right]}_{\triangleq \Phi(h, \tau)} \xi_{k}+\underbrace{\left[\begin{array}{c}
\int_{0}^{h-\tau} e^{A s} d s B \\
I_{m \times m}
\end{array}\right]}_{\triangleq \Gamma(h, \tau)} u_{k} \\
& +\underbrace{\left[\begin{array}{c}
I_{n \times n} \\
0_{m \times n}
\end{array}\right]}_{\triangleq G} v_{k} \\
& y_{k}=\rho_{k} \underbrace{\left[\begin{array}{ll}
\tilde{C} & 0_{1 \times m}
\end{array}\right]}_{\triangleq C} \xi_{k}+w_{k},
\end{aligned}
$$

where $v_{k}$ and $w_{k}$ are zero mean discrete-time Gaussian white noise process with

$$
\mathbf{E}\left\{\left[\begin{array}{c}
v_{k} \\
w_{k}
\end{array}\right]\left[\begin{array}{ll}
v_{k}^{\top} & w_{k}^{\top}
\end{array}\right]\right\}=\left[\begin{array}{cc}
R_{v} & 0 \\
0 & R_{w}
\end{array}\right]
$$

where $R_{v} \triangleq \int_{0}^{h} e^{A s} R_{v}^{c} e^{A^{\top} s} d s$.

Note that $u_{k}$ is not computed and applied until $t=k h+$ $\tau$, at which time $y_{k}$ is available to the controller unless it has been dropped by the network. Hence, the controller has access to the following information set when computing $u_{k}$ :

$$
\mathcal{I}_{k} \triangleq\left\{\mathcal{Y}_{k}, \mathcal{U}_{k-1}, \mathcal{R}_{k}\right\}
$$

where $\mathcal{Y}_{k}=\left(y_{k}, \ldots, y_{1}\right)$, and $\mathcal{U}_{k-1}=\left(u_{k-1}, \ldots, u_{1}\right)$, while $\mathcal{R}_{k}=\left(\rho_{k}, \ldots, \rho_{1}\right)$ is the realizations of the Bernoulli random variable $\rho_{k}$ that models successful packet transmissions.

1) Estimator Design: As in [10] the Kalman filter is the optimal estimator for our setting. The minimum mean square error (MMSE) estimate $\hat{\xi}_{k \mid k}$ of $\xi_{k}$ given by $\hat{\xi}_{k \mid k}=\mathbf{E}\left\{\xi_{k} \mid \mathcal{I}_{k}\right\}$ can be computed recursively starting from the initial conditions $\hat{\xi}_{0 \mid-1}=\operatorname{col}\left\{0_{n \times 1}, 0_{m \times 1}\right\}$ and $P_{0 \mid-1}=P_{0}$. The innovation step is

$$
\begin{aligned}
& \hat{\xi}_{k+1 \mid k} \triangleq \mathbf{E}\left\{\xi_{k+1} \mid \mathcal{I}_{k}\right\}=\Phi \hat{\xi}_{k \mid k}+\Gamma u_{k} \\
& e_{k+1 \mid k} \triangleq \xi_{k+1}-\hat{\xi}_{k+1 \mid k}=\Phi e_{k \mid k}+G v_{k} \\
& P_{k+1 \mid k} \triangleq \mathbf{E}\left\{e_{k+1 \mid k} e_{k+1 \mid k}^{\top} \mid \mathcal{I}_{k}\right\}=\Phi P_{k \mid k} \Phi^{\top}+\tilde{R}_{v}
\end{aligned}
$$

where $\tilde{R}_{v} \triangleq G R_{v} G^{\top}$ and $v_{k}$ is independent from $\mathcal{I}_{k}$, while the correction step is

$$
\begin{aligned}
\hat{\xi}_{k+1 \mid k+1} & =\hat{\xi}_{k+1 \mid k}+\rho_{k+1} K_{k+1}\left(z_{k+1}-C \hat{\xi}_{k+1 \mid k}\right) \\
e_{k+1 \mid k+1} & =\xi_{k+1}-\hat{\xi}_{k+1 \mid k+1} \\
P_{k+1 \mid k+1} & =P_{k+1 \mid k}-\rho_{k+1} K_{k+1} C P_{k+1 \mid k} \\
K_{k+1} & \triangleq P_{k+1 \mid k} C^{\top}\left(C P_{k+1 \mid k} C^{\top}+R_{w}\right)^{-1} .
\end{aligned}
$$

The following result, similar to [10], characterizes the estimation error covariance matrix. 
Proposition 4.1: The MMSE estimate $\hat{\xi}_{k \mid k}$ of $\xi_{k}$ is given by the time-varying Kalman filter (3) - (9). The expected value of the covariance matrix can be bounded as

$$
\underline{P}_{k \mid k} \leq \mathbf{E}_{\rho}\left\{P_{k \mid k}\right\} \leq \bar{P}_{k \mid k}
$$

where the bounds can be computed iteratively as

$$
\begin{aligned}
\bar{P}_{k+1 \mid k}= & \Phi \bar{P}_{k \mid k-1} \Phi^{\top}+\tilde{R}_{v}-\rho \Phi \bar{P}_{k \mid k-1} C^{\top} \\
& \times\left(C \bar{P}_{k \mid k-1} C^{\top}+R_{w}\right)^{-1} C \bar{P}_{k \mid k-1} \Phi^{\top} \\
\bar{P}_{k \mid k}= & \bar{P}_{k \mid k-1}-\rho \bar{P}_{k \mid k-1} C^{\top} \\
& \times\left(C \bar{P}_{k \mid k-1} C^{\top}+R_{w}\right)^{-1} C \bar{P}_{k \mid k-1} \\
\underline{P}_{k+1 \mid k}= & (1-\rho) \Phi \underline{P}_{k \mid k-1} \Phi^{\top}+\tilde{R}_{v} \\
\underline{P}_{k \mid k}= & (1-\rho) \underline{P}_{k \mid k-1}
\end{aligned}
$$

starting from the initial conditions $\underline{P}_{0 \mid-1}=\bar{P}_{0 \mid-1}=P_{0}$. When $k \rightarrow \infty$, the iterations converge to the unique stationary solutions $\underline{P}_{\infty}$ and $\bar{P}_{\infty}$ of the modified algebraic Riccati equations

$$
\begin{aligned}
\bar{P}_{\infty}= & \Phi \bar{P}_{\infty} \Phi^{\top}+\tilde{R}_{v} \\
& -\rho \Phi \bar{P}_{\infty} C^{\top}\left(C \bar{P}_{\infty} C^{\top}+R_{w}\right)^{-1} C \bar{P}_{\infty} \Phi^{\top} \\
\underline{P}_{\infty}= & (1-\rho) \Phi \underline{P}_{\infty} \Phi^{\top}+\tilde{R}_{v} .
\end{aligned}
$$

2) Controller Design: Next, we develop the optimal state feedback control law.

Proposition 4.2: Consider the aforementioned finite horizon LQG control problem. The optimal control law

$$
u_{k}=-\underbrace{\left(\Gamma^{\top} S_{k+1} \Gamma+\Xi_{u u}\right)^{-1}\left(\Gamma^{\top} S_{k+1} \Phi+\Xi_{\xi u}^{\top}\right)}_{L_{k}} \hat{\xi}_{k \mid k}
$$

is a linear function of the estimated state. The matrix $S_{k}$ evolves according to the backward Riccati recursion

$$
\begin{aligned}
S_{k}=\Phi^{\top} & S_{k+1} \Phi+\Xi_{\xi \xi}-\left(\Phi^{\top} S_{k+1} \Gamma+\Xi_{\xi u}\right) \\
& \times\left(\Gamma^{\top} S_{k+1} \Gamma+\Xi_{u u}\right)^{-1}\left(\Gamma^{\top} S_{k+1} \Phi+\Xi_{\xi u}^{\top}\right)
\end{aligned}
$$

where $\hat{\xi}_{k \mid k}$ is the MMSE estimate of the state $\xi_{k}$ based on the information set $\mathcal{I}_{k}$ computed with the Kalman filter (3) (9). As $k \rightarrow \infty$, the Riccati recursion converges to a unique stationary solution $S_{\infty}$ satisfying

$$
\begin{aligned}
S_{\infty}=\Phi^{\top} S_{\infty} \Phi & +\Xi_{\xi \xi}-\left(\Phi^{\top} S_{\infty} \Gamma+\Xi_{\xi u}\right) \\
& \times\left(\Gamma^{\top} S_{\infty} \Gamma+\Xi_{u u}\right)^{-1}\left(\Gamma^{T} S_{\infty} \Phi+\Xi_{\xi u}^{\top}\right)
\end{aligned}
$$

for which the associated stationary controller gain is

$$
L_{\infty} \triangleq \lim _{k \rightarrow \infty} L_{k}=-\left(\Gamma^{\top} S_{\infty} \Gamma+\Xi_{u u}\right)^{-1}\left(\Gamma^{\top} S_{\infty} \Phi+\Xi_{\xi u}^{\top}\right) .
$$

3) Optimal control cost: The loss function of the finite horizon LQG for the networked control system can be written as

$$
\begin{array}{r}
J_{N}^{\star}(\rho)=\xi_{0}^{\top} S_{0} \xi_{0}+\operatorname{Tr}\left(S_{0} P_{0}\right)+\sum_{k=0}^{N-1} \operatorname{Tr}\left(S_{k+1} \tilde{R}_{v}\right) \\
+\sum_{k=0}^{N-1} \operatorname{Tr}\left(\left(\Phi^{\top} S_{k+1} \Phi+\Xi_{\xi \xi}-S_{k}\right) \mathbf{E}_{\rho}\left\{P_{k \mid k}\right\}\right)
\end{array}
$$

where expectation is taken over a Bernoulli sequence $\left\{\rho_{k}\right\}$ with $\mathbf{E}\left\{\rho_{k}\right\}=\rho$. Since no efficient way of computing the expectation is known, one can use the upper and lower bounds on $\mathbf{E}_{\rho}\left\{P_{k \mid k}\right\}$ given in Proposition 4.1 to compute associated upper and lower bounds on the finite-horizon control cost $J_{N}^{\min }(\rho) \leq J_{N}^{\star}(\rho) \leq J_{N}^{\max }(\rho)$. For the infinite horizon case, the bounds become

$$
\begin{aligned}
J_{\infty}^{\min \triangleq} & \lim _{N \rightarrow \infty} \frac{1}{N} J_{N}^{\min } \\
= & \operatorname{Tr}\left(S_{\infty} \tilde{R}_{v}\right) \\
& +(1-\rho) \operatorname{Tr}\left(\left(\Phi^{\top} S_{\infty} \Phi+\Xi_{\xi \xi}-S_{\infty}\right) \underline{P}_{\infty}\right) \\
J_{\infty}^{\max \triangleq} & \lim _{N \rightarrow \infty} \frac{1}{N} J_{N}^{\max } \\
= & \operatorname{Tr}\left(S_{\infty} \tilde{R}_{v}\right)+\operatorname{Tr}\left(( \Phi ^ { \top } S _ { \infty } \Phi + \Xi _ { \xi \xi } - S _ { \infty } ) \left(\bar{P}_{\infty}\right.\right. \\
& \left.\left.-\rho \bar{P}_{\infty} C^{\top}\left(C \bar{P}_{\infty} C^{\top}+R_{w}\right)^{-1} C \bar{P}_{\infty}\right)\right)
\end{aligned}
$$

where the matrices $\underline{P}_{\infty}, \bar{P}_{\infty}$ and $S_{\infty}$, are given in Proposition 4.1 and Proposition 4.2, respectively.

To sum up, the optimal estimator is the time-varying Kalman filter given by (5), (8) and (9), while the optimal control is given by (12). The combined performance, in the sense of the continuous-time loss function (1), can be bounded as in (15) and (16). It is this controller and these performance bounds that we use in our co-design procedure.

\section{NeTWORK SUbPROBLEM}

In this section, we present the solution for the network subproblem of finding the policy that obtains the minimum energy cost and guarantees a probability that packets arrive within the sampling period (i.e., the packet deadline).

More specifically, we consider one sampling period scenario where a single packet, generated at time $t=0$, should be transmitted over a multi-hop wireless sensor network to the sink node $N$ within a deadline of $D \triangleq \frac{h}{t_{s}}$ time slots. Recall that $h$ is the sampling period and $t_{s}$ is the length of each time slot. The aim is to minimize the transmission energy cost $C(\pi)$ subject to the deadline-constrained reliability requirement $\rho_{\text {min }}(h)$. This minimum energy problem has recently been addressed in [11] with a general Markovian link loss model. We re-state the problem formulation and the main results for the independent packet losses in this paper.

\section{A. Constrained Markov decision process formulation}

The optimal forwarding problem can be formulated as a constrained Markov decision process (CMDP) [11].

The decision is made at time $t \in\{0,1, \ldots, D-1\}$. The state of the decision process $m_{t}$ denotes the packet location at time $t$. The action $j_{t}$ chooses the next hop node. The state transition probability $\operatorname{Pr}\left(m_{t+1} \mid m_{t}, j_{t}\right)$ is determined by link parameters. It has two cases depending on the action. If the action is to hold the packet, i.e., $j_{t}=m_{t}$, then

$$
\operatorname{Pr}\left(m_{t+1} \mid m_{t}, j_{t}\right)= \begin{cases}1 & \text { if } m_{t+1}=j_{t}, \\ 0 & \text { otherwise }\end{cases}
$$

If the action is to forward the packet, i.e., $j_{t} \neq m_{t}$, then

$$
\operatorname{Pr}\left(m_{t+1} \mid m_{t}, j_{t}\right)= \begin{cases}1-p_{m_{t} j_{t}} & \text { if } m_{t+1}=j_{t} \\ p_{m_{t} j_{t}} & \text { if } m_{t+1}=m_{t} \\ 0 & \text { otherwise. }\end{cases}
$$


The initial state is $m_{0}$ with the packet at the source node. There is a terminal reward $\mu\left(m_{D}\right)$ if the packet arrives at the sink node $N$ at the last time slot $D$,

$$
\mu\left(m_{D}\right)= \begin{cases}1 & \text { if } m_{D}=N \\ 0 & \text { otherwise }\end{cases}
$$

and a cost $c\left(m_{t}, j_{t}\right)$ when the action is to transmit the packet,

$$
c\left(m_{t}, j_{t}\right)= \begin{cases}\gamma & \text { if } j_{t} \neq m_{t} \\ 0 & \text { otherwise }\end{cases}
$$

where $\gamma$ is the energy cost for transmitting one packet.

Let history $h_{t}$ be a sequence of previous states and actions, i.e., $h_{t} \triangleq\left(m_{0}, j_{0}, \ldots, m_{t-1}, j_{t-1}, m_{t}\right)$, and $H_{t}$ be the set of all possible histories. The decision rule is a function $d_{t}: H_{t} \rightarrow \mathcal{P}\left(A_{t}\right)$ that maps $H_{t}$ into a set of probability distributions on the action space $A_{t}$ that includes all possible actions. A policy $\pi \triangleq\left(d_{0}, d_{1}, \ldots, d_{D-1}\right)$ is a time sequence of decision rules. Under a policy $\pi$, the expected reward (deadline-constrained packet reliability) is

$$
R(\pi) \triangleq \mathbf{E}_{m_{0}}^{\pi}\left\{\mu\left(m_{D}\right)\right\}
$$

and the expected energy cost is

$$
C(\pi) \triangleq \mathbf{E}_{m_{0}}^{\pi}\left\{\sum_{t=0}^{t=D-1} c\left(m_{t}, j_{t}\right)\right\}
$$

The minimum energy forwarding problem is

$$
\begin{array}{ll}
\underset{\pi}{\operatorname{minimize}} & C(\pi) \\
\text { subject to } & R(\pi) \geq \rho_{\min } .
\end{array}
$$

\section{B. Construction and structure of the optimal policy}

The Lagrange dual of the minimum energy problem (17) is

$$
\begin{array}{ll}
\underset{\lambda}{\operatorname{maximize}} & \min _{\pi}\left(C(\pi)+\lambda\left(\rho_{\min }-R(\pi)\right)\right) \\
\text { subject to } & \lambda \geq 0
\end{array}
$$

which is equivalent to

$$
\begin{array}{ll}
\underset{\delta}{\operatorname{maximize}} & \frac{1}{\delta} \max _{\pi}\{R(\pi)-\delta C(\pi)\}-\frac{\rho_{\min }}{\delta} \\
\text { subject to } & \delta \geq 0
\end{array}
$$

where $\delta=1 / \lambda$. By [12, Thm. 4.9], $R(\pi)$ and $C(\pi)$ are convex functions, and the duality gap is zero. To solve the problem (18), we hence need to study the weighted sum maximization of reliability and energy,

$$
\max _{\pi}\{R(\pi)-\delta C(\pi)\}
$$

for a given $\delta \geq 0$.

The weighted energy cost $\delta C(\pi)$ can be treated as negative rewards. This weighted sum maximization problem is then formulated as a Markov decision process. The optimal policy can be found by dynamic programming (DP). At time $t$ and node $i$, let the maximum utility be

$$
U_{i}^{\star}(t)=R_{i}^{\star}(t)-\delta C_{i}^{\star}(t) .
$$

The maximum utility $U_{i}^{\star}(0)$ along with the optimal policy are computed backwards by DP with the initial condition

$$
R_{i}^{\star}(D)=\left\{\begin{array}{ll}
1 & \text { if } i=N, \\
0 & \text { if } i \neq N ;
\end{array} \quad C_{i}^{\star}(D)=0 .\right.
$$

At each step with $t<D$, the maximum utility is

$$
U_{i}^{\star}(t)=\max \left\{\max _{j} U_{i}^{j}(t), U_{i}^{i}(t)\right\}
$$

where $U_{i}^{j}(t)$ is the utility of forwarding to neighbor $j$, and $U_{i}^{i}(t)$ is the utility of withholding the packet at node $i$, respectively. These utilities are computed as

$$
\begin{aligned}
U_{i}^{j}(t) & =\underbrace{q_{i j} U_{j}^{\star}(t+1)}_{\text {Success forward }}+\underbrace{\left(1-q_{i j}\right) U_{i}^{\star}(t+1)}_{\text {Fail forward }}-\underbrace{\delta \gamma}_{\text {Tx Cost }} ; \\
U_{i}^{i}(t) & =\underbrace{U_{i}^{\star}(t+1)}_{\text {Staying }} .
\end{aligned}
$$

Note the DP update requires the maximum utility $U_{i}^{\star}(t+1)$ computed at the previous time $t+1$, and also the maximum utility $U_{j}^{\star}(t+1)$ from node $j$ by a similar DP algorithm.

The optimal action at time $t$ when node $i$ holds the packet is to forward to the node that maximizes Eq. (19). The optimal policy for the weighted sum maximization with a given $\delta$ is composed by the optimal action at each time and node and is found along the DP backward computations. With the optimal policy, the corresponding reliability $R^{\star}(0)$ and energy cost $C^{\star}(0)$ can be readily computed.

Define $\mathcal{R}$ as the set of all possible $R^{\star}(0)$ that can be obtained by sweeping over values of $\delta$ in the dynamic programming. We can obtain an optimal policy for the minimum energy problem as follows.

Theorem 5.1 [11]: Let $R^{(1)}=\sup \left\{R \in \mathcal{R}: R \leq \rho_{\min }\right\}$ and $R^{(2)}=\inf \left\{R \in \mathcal{R}: R>\rho_{\min }\right\}$ with associated energy costs $C^{(1)}$ and $C^{(2)}$. The optimal value of the minimum energy forwarding problem (17) is then

$$
C^{\star}=C^{(1)}+\frac{\rho_{\min }-R^{(1)}}{R^{(2)}-R^{(1)}}\left(C^{(2)}-C^{(1)}\right) .
$$

Suppose optimal policies that can obtain $R^{(1)}, C^{(1)}$ and $R^{(2)}, C^{(2)}$ are $\pi^{(1)}$ and $\pi^{(2)}$ respectively. An optimal policy $\pi^{\star}$ for the minimum energy problem is obtained by random selection of policies $\pi^{(1)}$ and $\pi^{(2)}$ with probabilities

$$
\theta^{(1)}=\frac{R^{(2)}-\rho_{\min }}{R^{(2)}-R^{(1)}} ; \quad \theta^{(2)}=\frac{\rho_{\min }-R^{(1)}}{R^{(2)}-R^{(1)}} .
$$

\section{NUMERICAL EXAMPLES}

We are now ready to demonstrate the co-design procedure on a numerical example. Consider the process

$$
\begin{aligned}
d x & =\left[\begin{array}{cc}
0 & 1 \\
-1 & 2
\end{array}\right] x d t+\left[\begin{array}{l}
0 \\
1
\end{array}\right] u d t+d v_{c}, \\
y(k h) & =\left[\begin{array}{ll}
1 & 0
\end{array}\right] x(k h)+w(k h),
\end{aligned}
$$

where $v_{c}$ has incremental covariance $R_{v}^{c}=\operatorname{diag}\{1,0.5\}$ and $R_{w}=10^{-4}$, and the linear-quadratic control loss in (1) with $Q_{x x}^{c}=\operatorname{diag}\{2,5\}, Q_{x u}^{c}=0_{2 \times 1}, Q_{u u}^{c}=1$, and $Q_{0}^{c}=0_{2 \times 2}$. 


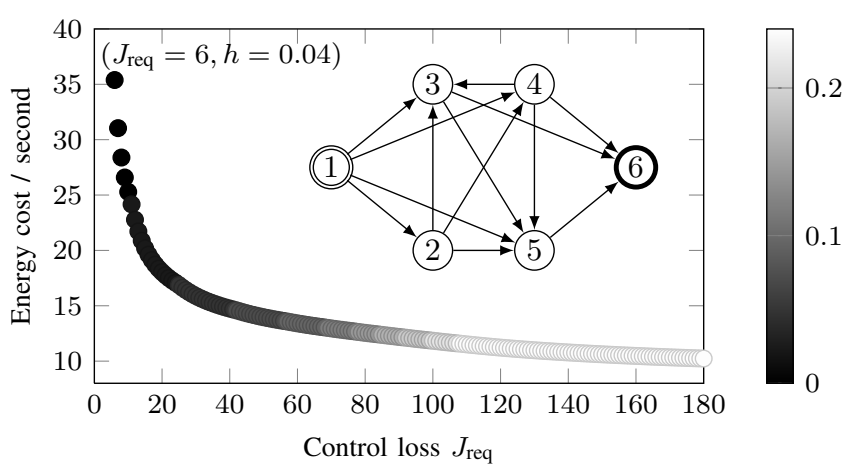

Fig. 2. The minimum energy cost for different control loss requirements and the corresponding optimal sampling periods (shown in gray scale).

Moreover, the optimal control loss is measured by the upper bound for the infinite horizon case.

The topology of the wireless network between sensor and controller is shown in Fig. 2 where node 1 is the source and node 6 is the sink. The loss probability on each link is randomly chosen between 0.1 and 0.8 . Each time slot is 0.02 seconds long. We assume the transmission energy cost is the same and fixed for all nodes, and normalize this value to 1 . Fig. 2 displays the minimum energy cost for a range of control performance requirements. The minimum energy cost naturally increases when the control performance requirement becomes increasingly stringent. Moreover, the optimal sampling periods are shown in gray scale with shorter periods for smaller control loss. The minimum sampling period required to obtain a finite control loss is $40 \mathrm{~ms}$ which corresponds to the minimum hop count of two in the wireless network. The associated optimal control cost is six. An interesting observation is that it is very costly to obtain the minimal control loss, and that significant energy savings can be obtained by accepting a relatively small deterioration in the control performance. Fig. 3 shows the minimum energy cost for different sampling periods. For a given control performance requirement, the relation between sampling period and energy cost is neither monotone decreasing nor monotone increasing except for very lax control performance requirements. This necessitates the procedure of sweeping all sampling periods to obtain the minimum energy.

\section{CONCLUSION}

We have studied the minimum-energy packet forwarding policies for a guaranteed closed-loop control performance in wireless control systems in which the sensor measurements are sent from plant to controller over an unreliable multi-hop network. Based on observation that for fixed sampling period the minimal control loss is monotonically decreasing in the end-to-end reliability, we propose an optimal decomposition of the problem into two subproblems. The minimum endto-end reliability that allows to guarantee a target control performance can be found by a bisection algorithm, and the network subproblem of minimizing the energy cost subject to a deadline-constrained reliability can be solved as a

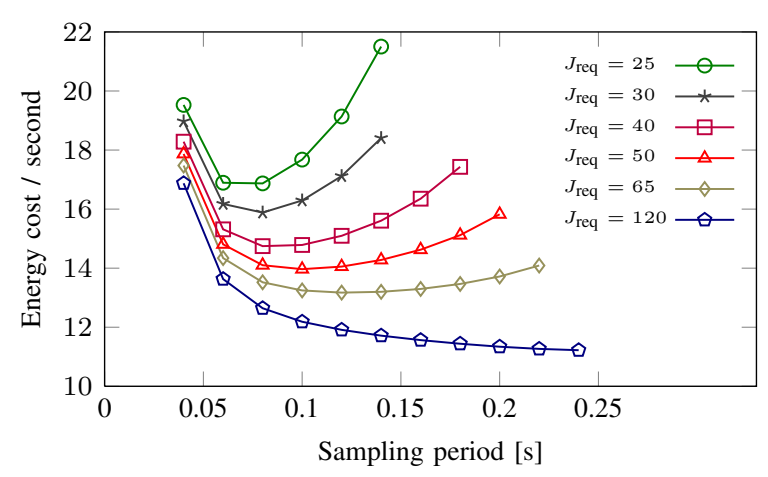

Fig. 3. The minimum energy cost for different sampling periods.

constrained Markov decision process. The jointly optimal design is found by a search over sampling periods.

A natural extension would be to consider link-losses that are correlated in time. The CMDP approach can readily handle the case when link-losses are described by finite-state Markov chains as long as the link states are known (possibly with a delay). Extensions to scheduling with partial channel state information are also possible. However, when the endto-end losses are correlated in time, it appears hard to design optimal controllers that do not require full network state information, and modular co-design remains challenging.

\section{REFERENCES}

[1] G. Walsh, H. Ye, and L. Bushnell, "Stability analysis of networked control systems," IEEE Transactions on Control Systems Technology, vol. 10, no. 3, pp. 438-446, 2002.

[2] B. Demirel, Z. Zou, P. Soldati, and M. Johansson, "Modular co-design of controllers and transmission schedules in WirelessHART," in Proc. IEEE CDC, 2011.

[3] M. Zafer and E. Modiano, "Minimum energy transmission over a wireless channel with deadline and power constraints," IEEE Transactions on Automatic Control, vol. 54, no. 12, pp. 2841-2852, 2009.

[4] Y. Gu, T. He, M. Lin, and J. Xu, "Spatiotemporal delay control for low-duty-cycle sensor networks," in Proc. RTSS, 2009, pp. 127-137.

[5] R. Cohen and B. Kapchits, "An optimal wake-up scheduling algorithm for minimizing energy consumption while limiting maximum delay in a mesh sensor network," IEEE/ACM Transactions on Networking, vol. 17 , no. 2, pp. 570-581, 2009.

[6] B. Demirel, Z. Zou, P. Soldati, and M. Johansson, "Modular design of jointly optimal controllers and forwarding policies for wireless control," 2012, arXiv:1204.3100v1.

[7] L. Shi, K. H. Johansson, and R. M. Murray, "Change sensor topology when needed:How to efficiently use system resources in control and estimation over wireless networks," in Proc. IEEE CDC, 2007.

[8] Y. Mo, E. Garone, A. Casavola, and B. Sinopoli, "Stochastic sensor scheduling for energy constrained estimation in multi-hop wireless sensor networks," IEEE Transactions on Automatic Control, vol. 56, no. 10, pp. 2489-2495, 2011.

[9] P. Park, J. Araujo, and K. Johansson, "Wireless networked control system co-design," in Proc. ICNSC, 2011, pp. 486-491.

[10] L. Schenato, B. Sinopoli, M. Francescchetti, K. Poolla, and S. Sastry, "Foundatiolns of control and estimation over lossy networks," Proceedings of the IEEE, vol. 95, no. 1, pp. 163-187, 2007.

[11] Z. Zou and M. Johansson, "Minimum-energy packet forwarding over lossy networks under deadline and reliability constraints," in Proc. WiOpt, 2012, pp. 224-231.

[12] E. Altman, Constrained Markov Decision Processes. Chapman and Hall/CRC, 1999. 